\title{
A One-Sided Version of the Poisson Sum Formula for Semi-Infinite Array Green's Functions
}

\author{
J. Paul Skinner and Peter J. Collins, Student Member, IEEE
}

\begin{abstract}
The Poisson sum formula provides an efficient method for transforming many slowly converging infinite summations into equivalent, but more rapidly converging infinite summations. Electromagnetic applications for this result come in the analysis of infinite arrays of periodic scatterers, such as frequency selective surfaces. However, in some applications, such as when one desires the radiation of a semi-infinite array of periodically spaced currents, the original form of the Poisson sum formula is inappropriate. For such applications, we derive a one-sided version of the formula and apply it to the radiation from a semi-infinite array of line sources with currents dictated by Floquet's theorem. The one-sided Poisson sum transformation yields enhanced convergence characteristics for certain regions of application as a result of the inverse bandwidth relationship between Fourier transform pairs. The application of it to a semi-infinite line source array also provides a plane wave representation for the fields, which makes for an extension of the solution to geometries with stratified dielectric media.
\end{abstract}

Index Terms-Arrays.

\section{INTRODUCTION}

$\mathbf{T}$ HIS paper presents the derivation of a modified version of the Poisson sum formula, which can be used on certain one-sided summations to possibly enhance numerical convergence. In this paper, we present an application of the formula to a semi-infinite array Green's function. This Green's function is based on regularly spaced line sources that have equivalent amplitudes on each filament, but with a progressive phase between elements in accordance with Floquet's theorem.

We recognize that a semi-infinite array will normally tend to have different current amplitudes and phases than the Floquet assumption for the elements near the edge of the array. However, in using a Floquet assumption we are dealing with a semi-infinite array of elements that is a subset of a "true" semi-infinite array, i.e., the true array minus the nonuniform edge elements. The Floquet based currents can be used as a semi-infinite basis function which, along with single element basis functions near the true edge of the array, can be used in a moment method analysis.

Felsen and Carin have investigated finite arrays using Floquet current approximations [1], [2]. They do not, however, derive or use the one-sided Poisson sum formula that we present here. The key distinction between their work and ours lies in the application. Our expressions are valid for observa-

Manuscript received December 15, 1995; revised June 7, 1996.

The authors are with the Department of Electrical and Computer Engineering, Air Force Institute of Technology, AFIT/ENG, Wright-Patterson Air Force Base, $\mathrm{OH} 45433$ USA.

Publisher Item Identifier S 0018-926X(97)02493-9. tion points arbitrarily close to the array plane. We specifically developed them to calculate the mutual coupling between a semi-infinite array basis function and a nearby singleedge element basis function. In contrast, Felsen and Carin present "... a theory of diffraction that deals with scatteredfield phenomena away from the grating plane ..." [1].

The remainder of this paper is divided into sections outlining the derivation of the modified version of Poisson's sum formula, the application to the semi-infinite array of line sources, the results of the semi-infinite array Green's function, and a brief conclusion section.

\section{Derivation of a One-Sided Poisson Sum Formula}

Using the Fourier transform pair

$$
\begin{aligned}
f(t) & =\frac{1}{2 \pi} \int_{-\infty}^{\infty} F(\omega) e^{j \omega t} d \omega \\
F(\omega) & =\int_{-\infty}^{\infty} f(t) e^{-j \omega t} d t
\end{aligned}
$$

the ordinary Poisson sum formula is [3]

$$
\sum_{m=-\infty}^{\infty} e^{j m \omega_{0} t} F\left(m \omega_{0}\right)=T \sum_{n=-\infty}^{\infty} f(t \pm n T)
$$

where $\omega_{0}=\frac{2 \pi}{T}$. It is obvious by the symmetry of the limits in the summation that either the plus or minus in (3) may be used as long as the chosen sign is consistently applied. We desire a one-sided form where the summation on the lefthand side of (3) has the limits from $m=0$ to $m=\infty$. To accomplish this, we simply define $F(\omega)$ to be the product of an infinite domain continuous function $G(\omega)$ and the Heaviside unit step function shifted by $\frac{\omega_{o}}{2}$ to "capture" the entire $m=0$ term. Using the appropriate "generalized" Fourier transforms and properties [5], [6], we can express the inverse Fourier transform of $F(\omega)$ as

$$
f(t)=\frac{g(t)}{2}+\frac{j}{2 \pi} f_{-\infty}^{\infty} \frac{g(\tau) e^{-j \frac{\omega_{0}}{2}(t-\tau)}}{t-\tau} d \tau .
$$

Substituting these expressions back into (3), we get a one-sided version of the Poisson sum formula

$$
\begin{aligned}
& \sum_{m=0}^{\infty} e^{j m \omega_{0} t} G\left(m \omega_{0}\right) \\
& =T \sum_{n=-\infty}^{\infty}\left\{\frac{g(t \pm n T)}{2}+\frac{j}{2 \pi} f_{-\infty}^{\infty} \frac{g(\tau) e^{-j \frac{\omega_{0}}{2}(t \pm n T-\tau)}}{t \pm n T-\tau} d \tau\right\} .
\end{aligned}
$$

U.S. Government work not protected by U.S. copyright. 


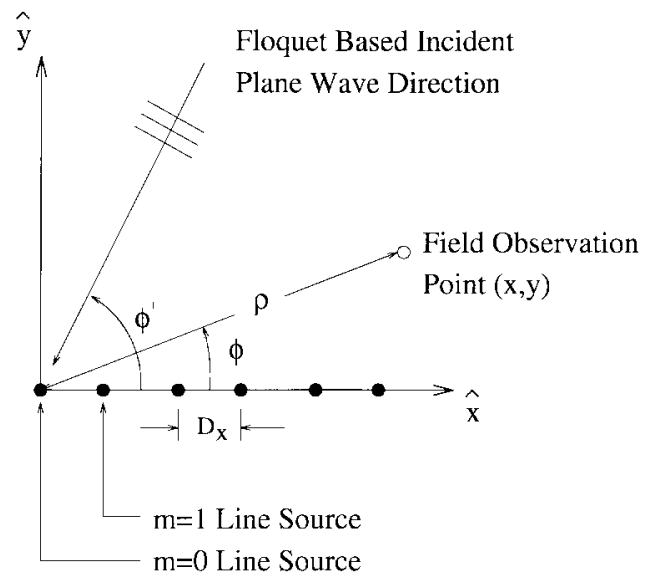

Fig. 1. Cross-sectional view of a semi-infinite array of line sources.

Again, either the plus signs or minus signs may be chosen, but the same choice must be taken in each circumstance, and for each value of $n$.

\section{Application to a Semi-Infinite ARRAY OF LINE SOURCES}

Consider a semi-infinite array of $\hat{z}$ directed line sources, with regular spacing $D_{x}$ along the positive half of the $x$ axis. Fig. 1 gives a cross-sectional view of the array, along with coordinate definitions for a field-observation point and a hypothetical plane wave propagation direction associated with the Floquet phase factors to come.

We may spatially index the line sources such that $m=0$ is the first line source (at the origin), and subsequent line sources are associated with increasing positive integer values of $m$. Even though the array is semi-infinite, we will assume that we are dealing only with a subset of elements, which is also semi-infinite, but is electrically removed from the edge of the true semi-infinite array. Thus, we assume the currents obey Floquet's theorem [7], so that the current on the $m$ th element is

$$
J_{m}=J_{0} e^{j \beta m D_{x} \cos \phi^{\prime}}
$$

with $J_{0}$ being the current on the edge element. We again remind the reader that the perturbation of the currents near the true edge of a semi-infinite array can be handled separately from this analysis. A semi-infinite array free-space Green's function can thus be formed by the superposition of the radiation of each individual line source. We can express the spatial sum representation of this Green's function as

$$
\mathcal{G}(x, y)=-\frac{j}{4} \sum_{m=0}^{\infty} H_{0}^{(2)}\left(\beta \sqrt{\left(x-m D_{x}\right)^{2}+y^{2}}\right) e^{j \beta m D_{x} \cos \phi^{\prime}} .
$$

We will apply this to the one-sided Poisson sum formula with the spatial-domain summation corresponding to the left-hand side of (5). To do this, we use the Fourier transform pair [8]

$$
g(t)=\frac{e^{j x t} e^{-j y \sqrt{\beta^{2}-t^{2}}}}{\pi \sqrt{\beta^{2}-t^{2}}} \leftrightarrow G(\omega)=H_{0}^{(2)}\left(\beta \sqrt{(x-\omega)^{2}+y^{2}}\right) .
$$

Through a careful substitution of variables, (5) can then be used to transform the semi-infinite array free-space Green's function into

$$
\mathcal{G}(x, y)=-\frac{j}{4 \pi} \sum_{n=-\infty}^{\infty}\left\{\frac{e^{-j\left(x \alpha_{n}+y \sqrt{\beta^{2}-\alpha_{n}^{2}}\right)}}{\sqrt{\beta^{2}-\alpha_{n}^{2}}}-\frac{j}{\pi} I_{n}\right\}
$$

where

$$
I_{n}=f_{-\infty}^{\infty} \frac{e^{j\left(x \zeta-y \sqrt{\beta^{2}-\zeta^{2}}\right)} e^{j \frac{D_{x}}{2}\left(\alpha_{n}+\zeta\right)}}{\sqrt{\beta^{2}-\zeta^{2}}\left(\alpha_{n}+\zeta\right)} d \zeta
$$

and

$$
\alpha_{n}=\frac{2 \pi n}{D_{x}}-\beta \cos \phi^{\prime}
$$

We refer to (9) as being a spectral-domain summation for the Green's function.

The first term in the spectral summation in (9) represents a discrete bundle of plane waves, which is similar to the plane wave spectrum generated by fully infinite arrays with Floquet governed currents [9]. The direction of propagation for the $n$th plane wave is

$$
\hat{r}_{n}=\hat{x} \frac{\alpha_{n}}{\beta}+\hat{y} \frac{\sqrt{\beta^{2}-\alpha_{n}^{2}}}{\beta} .
$$

Without loss of generality, we confine the value of $y$ to be nonnegative and, thus, the square roots in (9) through (12) must be chosen with either the positive real or " $-j$ " root, to obey the radiation condition. A finite number of terms clustered around $n=0$ will yield $\left|\alpha_{n}\right|<\beta$, which implies a propagating plane wave. The other plane waves are evanescent and decay rapidly as $y$ is increased in value.

The second term in (9) represents a continuous spectrum of plane waves generated by truncating the infinite wire array. Its form should be recognizable to those familiar with edge diffraction from a half plane. We will use this similarity to address the difficulties encountered in evaluating the principal value integral, in particular, the branch points at $\zeta= \pm \beta$. For integrals of this type, Felsen [10] suggests the variable substitution

$$
\zeta=-\beta \sin \nu
$$

When we employ this substitution, we obtain an angular spectral-integral representation devoid of the branch point singularities

$$
I_{n}=e^{j \frac{D_{x}}{2} \alpha_{n}} f_{C} \frac{e^{-j \beta\left[\left(x+\frac{D_{x}}{2}\right) \sin \nu+y \cos \nu\right]}}{\left(\beta \sin \nu-\alpha_{n}\right)} d \nu .
$$

We note that the proper branch of the arcsin must be used to define the integration contour $C$ to satisfy the radiation condition [10]. The contour $C$ in the complex $\nu$ plane contains one pole at $\nu=\nu_{0}=\sin ^{-1}\left(\frac{\alpha_{n}}{\beta}\right)$, related to the $n t h$ spectral term.

Consider a displaced circular-cylindrical coordinate system, with an origin at $x=-\frac{D_{x}}{2}, y=0$. From it, we may describe a point by the position $(\tilde{\rho}, \tilde{\phi})$, where

$$
\tilde{\rho}=\sqrt{\left(x+\frac{D_{x}}{2}\right)^{2}+y^{2}}
$$




$$
\tilde{\phi}=\arctan \left[\frac{x+\frac{D_{x}}{2}}{y}\right] .
$$

Note that $\tilde{\phi}$ designates an angle at the displaced origin relative to the normal from the array. Using these coordinates and employing the cosine addition formula, we write the angular spectral integral as

$$
I_{n}=e^{j \frac{D_{x}}{2} \alpha_{n \imath}} f_{C} \frac{e^{-j \beta \tilde{\rho} \cos (\nu-\tilde{\phi})}}{\left(\beta \sin \nu-\alpha_{n}\right)} d \nu
$$

The angular spectral integral in (17) may be evaluated via numerical integration or, preferably, using a closed-form expression obtained from asymptotic analysis. When numerical integration is required, the singularity may be extracted analytically, leaving a smooth integrand for the numerical sampling. To accomplish this regularization, we divide the total integral associated with the contour $C$ into the sum $I_{n}=I_{n 1}+I_{n 2}+I_{n 3}$, where the limits for $I_{n 1}$ run from $-\frac{\pi}{2}-j \infty$ to $-\frac{\pi}{2}$, the limits for $I_{n 2}$ run from $-\frac{\pi}{2}$ to $\frac{\pi}{2}$, and the limits for $I_{n 3}$ run from $\frac{\pi}{2}$ to $\frac{\pi}{2}+j \infty$. We may transform paths in $I_{n 1}$ and $I_{n 3}$ to the real axis and extract the singularity where appropriate. For the first integral, this results in

$$
\begin{aligned}
I_{n 1}= & e^{j \frac{D_{x}}{2} \alpha_{n}} f_{-\frac{\pi}{2}-j \infty}^{-\frac{\pi}{2}} \frac{e^{-j \beta \tilde{\rho} \cos (\nu-\tilde{\phi})}}{\beta \sin \nu-\alpha_{n}} d \nu \\
=-j e^{j \frac{D_{x}}{2} \alpha_{n}} & f_{0}^{\infty} \frac{e^{j \beta \tilde{\rho} \sin (j \nu+\tilde{\phi})}}{\beta \cosh \nu+\alpha_{n}} d \nu \\
=-j e^{j \frac{D_{x}}{2} \alpha_{n}} & \left\{\int_{0}^{\infty} \frac{e^{j \beta \tilde{\rho} \sin (j \nu+\tilde{\phi})}-e^{j \beta \tilde{\rho} \sin \left(j \nu_{0}+\tilde{\phi}\right)}}{\beta \cosh \nu+\alpha_{n}} d \nu\right. \\
& \left.+e^{j \beta \tilde{\rho} \sin \left(j \nu_{0}+\tilde{\phi}\right)} f_{0}^{\infty} \frac{d \nu}{\beta \cosh \nu+\alpha_{n}}\right\} .
\end{aligned}
$$

The nonprincipal value integral in (18) may appear to have a pole at $\nu=\nu_{0}$, but instead behaves smoothly in the neighborhood of this point. Thus, numerical integration may be successfully implemented. The last (principal value) integral in (18) still contains the pole, but it is of a form with a known evaluation. From [11], we get

$$
\begin{aligned}
& f_{0}^{\infty} \frac{d \nu}{\beta \cosh \nu+\alpha_{n}} \\
& = \begin{cases}\frac{2}{\sqrt{\beta^{2}-\alpha_{n}^{2}}} \arctan \left[\frac{\sqrt{\beta^{2}-\alpha_{n}^{2}}}{\beta+\alpha_{n}}\right], & \alpha_{n}^{2}<\beta^{2} \\
\frac{1}{\sqrt{\alpha_{n}^{2}-\beta^{2}}} \ln \left|\frac{\beta+\alpha_{n}}{\beta+\sqrt{\alpha_{n}^{2}-\beta^{2}}}\right|, \sqrt{\alpha_{n}^{2}-\beta^{2}} \mid, & \alpha_{n}^{2}>\beta^{2} .\end{cases}
\end{aligned}
$$

The regularization of the integrand in $I_{n 3}$ is handled in a similar manner, as used for $I_{n 1}$. The result is

$$
\begin{aligned}
I_{n 3}=j e^{j \frac{D_{x}}{2} \alpha_{n}}\{ & \int_{0}^{\infty} \frac{e^{j \beta \tilde{\rho} \sin (j \nu-\tilde{\phi})}-e^{j \beta \tilde{\rho} \sin \left(j \nu_{0}-\tilde{\phi}\right)}}{\beta \cosh \nu-\alpha_{n}} d \nu \\
& \left.+e^{j \beta \tilde{\rho} \sin \left(j \nu_{0}-\tilde{\phi}\right)} f_{0}^{\infty} \frac{d \nu}{\beta \cosh \nu-\alpha_{n}}\right\} .
\end{aligned}
$$

The principal value integral in (20) may be evaluated using (19) with the substitution of $-\alpha_{n}$ for $\alpha_{n}$. Finally, the $I_{n 2}$ integral is regularized without a change in integration path,

$$
\begin{aligned}
& \text { giving } \\
& \qquad \begin{array}{r}
I_{n 2}=e^{j \frac{D_{x}}{2} \alpha_{n}}\left\{\int_{-\frac{\pi}{2}}^{\frac{\pi}{2}} \frac{e^{-j \beta \tilde{\rho} \cos (\nu-\tilde{\phi})}-e^{-j \beta \tilde{\rho} \cos \left(\nu_{0}-\tilde{\phi}\right)}}{\beta \sin \nu-\alpha_{n}} d \nu\right. \\
\left.+e^{-j \beta \tilde{\rho} \cos \left(\nu_{0}-\tilde{\phi}\right)} f_{-\frac{\pi}{2}}^{\frac{\pi}{2}} \frac{d \nu}{\beta \sin \nu-\alpha_{n}}\right\}
\end{array}
\end{aligned}
$$

where, from [11], we get

$f_{-\frac{\pi}{2}}^{\frac{\pi}{2}} \frac{d \nu}{\beta \sin \nu-\alpha_{n}}=$

$\begin{cases}0, & \frac{2}{\sqrt{\alpha_{n}^{2}-\beta^{2}}}\left[\arctan \left(\frac{\beta-\alpha_{n}}{\sqrt{\alpha_{n}^{2}-\beta^{2}}}\right)-\arctan \left(\frac{\beta+\alpha_{n}}{\sqrt{\alpha_{n}^{2}-\beta^{2}}}\right)\right], \\ \alpha_{n}^{2}<\beta^{2} & \alpha_{n}^{2}>\beta^{2} .\end{cases}$

Finally, we note that while regularization is not strictly required when the singularity does not lie along the integration path of $I_{n 1}, I_{n 2}$, or $I_{n 3}$ (whichever one is being evaluated), it is numerically expedient to perform the extraction as long as the pole is near one of the endpoints of the path involved.

In the majority of cases, the previous numerical evaluation may be avoided by an asymptotic evaluation of the angular spectral integral using the method of steepest descent. The integral form in (17) is like a canonical form presented by Felsen [10]. The form contains a saddle point at $\nu=\nu_{s}=\tilde{\phi}$, which lies between $-\frac{\pi}{2}$ and $\frac{\pi}{2}$. The steepest descent path (SDP) and the contour $C$ intersect at the saddle point and at $\nu=\frac{\pi}{2}+j \nu_{c}$ where

$$
\nu_{c}=\ln \left[\frac{1+\cos \tilde{\phi}}{|\sin \tilde{\phi}|}\right] .
$$

When $\tilde{\phi}<0$, the contours intersect at the saddle point and at $\nu=-\frac{\pi}{2}-j \nu_{c}$.

Using $\beta \tilde{\rho}$ as a large parameter, the method of steepest descent can give a uniform asymptotic evaluation, valid even when the pole and saddle point become arbitrarily close. The SDP integral is then related to the desired principal value integral through a half residue contribution. The result is

$$
I_{n} \sim I_{S D P}+I_{\epsilon}
$$

where $I_{S D P}$ in (25) is shown at the bottom of the next page, where

$$
Q(x)=\int_{x}^{\infty} e^{-t^{2}} d t
$$

Note that $Q$ is related to the complementary error function. When its argument is complex, the path of integration is any path which starts at this complex value, and ends at $R$ where $|R| \rightarrow \infty$ and $-\frac{\pi}{4}<\arg (R)<\frac{\pi}{4}$. Information to get an evaluation of $Q$ for complex arguments without resorting to numerical integration is provided in [12]. The upper signs in (25) are chosen when the pole lies to the left of the SDP in the complex $\nu$ plane. The necessary conditions for this to occur are:

1) $\tilde{\phi}>0$ and $\operatorname{Re}\left(\nu_{0}\right)<\tilde{\phi}$;

2) $\tilde{\phi}>0$ and $\operatorname{Im}\left(\nu_{0}\right)_{\sim}>\nu_{c}$ or;

3) $\tilde{\phi}<0, \operatorname{Re}\left(\nu_{0}\right)<\tilde{\phi}$ and $\operatorname{Im}\left(\nu_{0}\right)>-\nu_{c}$. 


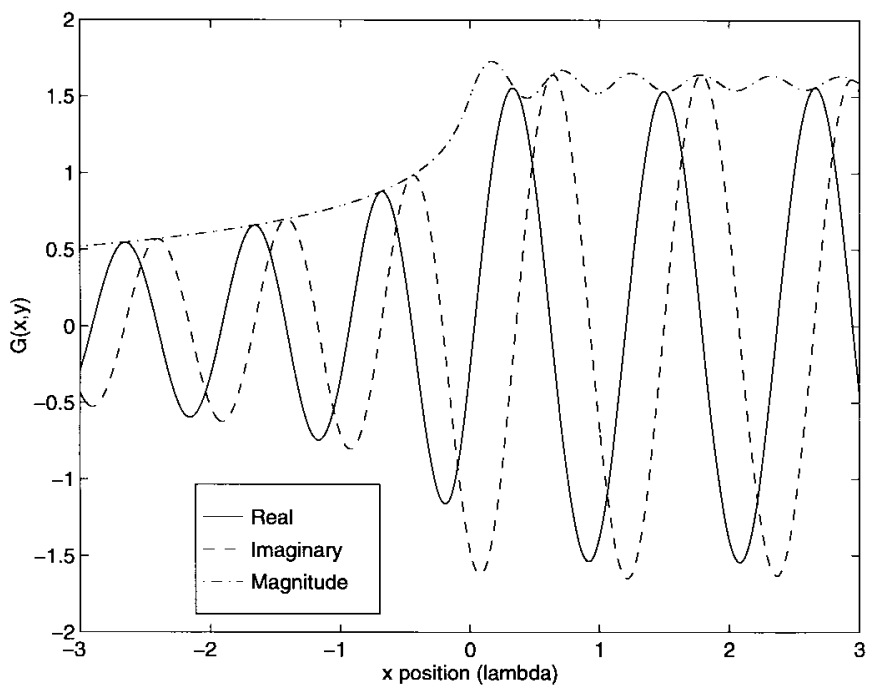

Fig. 2. Semi-infinite array green's function for $D_{x}=0.1 \lambda, \phi^{\prime}=30^{\circ}$ along a path of $-3 \lambda<x<+3 \lambda$ at $y=0.1 \lambda$.

Failure to satisfy at least one of the above three conditions implies the pole is not to the left of the SDP, in which case the lower signs in (25) must be chosen.

The half residue contribution is

$$
I_{\epsilon}= \pm j \pi \frac{e^{j \frac{D_{y}}{2} \alpha_{n}} e^{-j \beta \tilde{\rho} \cos \left(\nu_{0}-\tilde{\phi}\right)}}{\sqrt{\beta^{2}-\alpha_{n}^{2}}}
$$

where the plus sign corresponds to when the pole is to the right of the SDP, and the minus sign corresponds to the pole lying to the left of the SDP. The flip in signs here, as well as in $I_{S D P}$, are necessary to produce field continuity. This is very similar to the case of plane wave diffraction from a half-plane as one sweeps the observation point in angle $(\tilde{\phi})$ through a "shadow boundary." For example, when we consider the $n=0$ terms in the spectral sum, the pole defines a "shadow boundary" which is crossed at $\tilde{\phi}=\phi^{\prime}-\frac{\pi}{2}$, where $\phi^{\prime}$ is associated with the Floquet phase factors, as shown in Fig. 1.

\section{Results of the SEMi-Infinite ARRAY GREEN'S FUNCTION}

This section provides some numerical results of the Green's function described by either the spatial sum in (7) or the spectral sum in (9). A substantial number of numerical tests could be generated, but for the sake of brevity, we only present results related to a certain typical test case. This case is defined by an interelement spacing of $D_{x}=0.1 \lambda$ with the Floquet phasing associated with $\phi^{\prime}=30^{\circ}$ (see Fig. 1). With the exception of the case in Fig. 5, we have

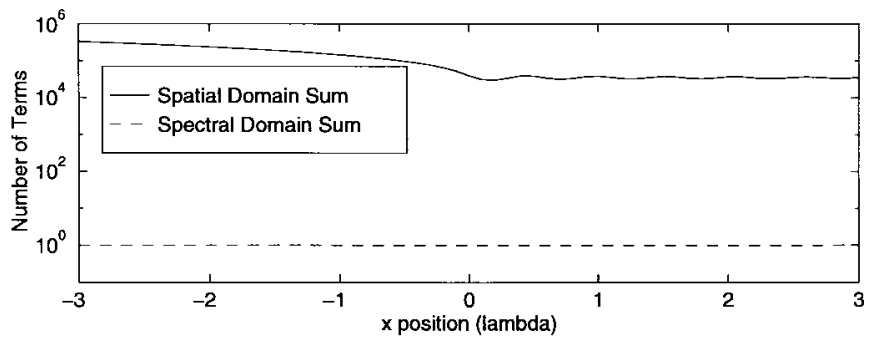

(a)

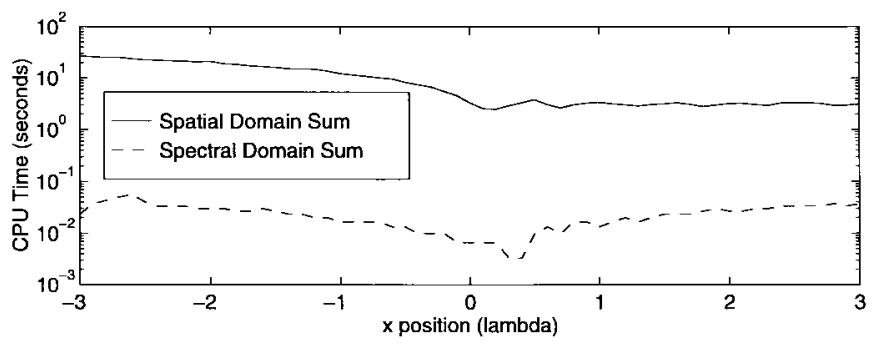

(b)

Fig. 3. (a) Terms required. (b) CPU time required for $1.0 \%$ convergence using unaccelerated spatial sum and unaccelerated spectral sum $D_{x}=0.1 \lambda, \phi^{\prime}=30^{\circ}, y=0.1 \lambda$.

limited our observation of the radiated field to a path from $-3 \lambda<x<+3 \lambda$ at a fixed "elevation" of $y=0.1 \lambda$. Thus, half of this path is directly above the semi-infinite array, where the other half is past the array edge.

Fig. 2 depicts the evaluation of the semi-infinite array Green's function along the observation path. Note that the field magnitude decays past the edge of the array. It also asymptotically approaches the value which it would have from the radiation of a full infinite array as $x \rightarrow+\infty$. For large positive values of $x$, the continuous spectrum term of (9) is almost identical to the discrete spectrum term. In fact, we should expect this to happen as the discrete spectrum term is exactly half the value one would get from the radiation of a full infinite array, as one can see by comparing the first term on the right-hand side of (5) with the right-hand side of (3).

The data generated for Fig. 2 can come from either the spatial (7) or spectral sums (9). The natural question is, "Which representation is more efficient?" There are two components of efficiency to consider. For the subject summations, these components are: 1) the number of terms required for the summation to converge and 2) the computation burden associated with calculating these terms. In the following examples, the spectral-domain terms are computed using the numerical integration approach described in the previous section. Consequently, the computation times represent a "worse"

$$
\begin{aligned}
I_{S D P} \sim e^{j \frac{D_{x}}{2} \alpha_{n}} e^{-j \beta \tilde{\rho}} \cdot\left\{e^{j \frac{\pi}{4}} \sqrt{\frac{2 \pi}{\beta \tilde{\rho}}}\left[\frac{1}{\beta \sin \tilde{\phi}-\alpha_{n}}-\frac{j}{\sqrt{\beta^{2}-\alpha_{n}^{2}} \sqrt{2\left[\cos \left(\nu_{0}-\tilde{\phi}\right)-1\right]}}\right]\right. \\
\quad \pm j 2 \sqrt{\frac{\pi}{\beta^{2}-\alpha_{n}^{2}}} e^{-j \beta \tilde{\rho}\left(\cos \left(\nu_{0}-\tilde{\phi}\right)-1\right)} Q\left(\mp e^{j \frac{3 \pi}{4}} \sqrt{\left.\beta \tilde{\rho}\left(\cos \left(\nu_{0}-\tilde{\phi}\right)-1\right)\right)}\right\}
\end{aligned}
$$




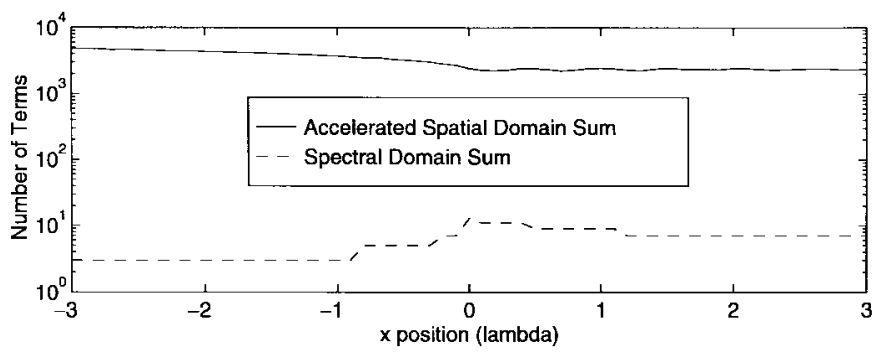

(a)

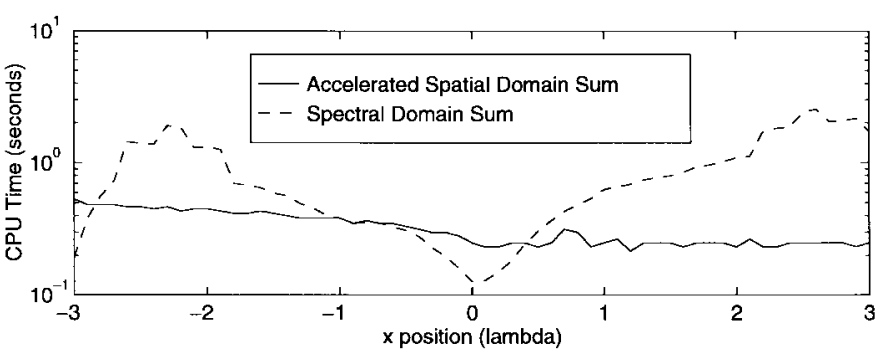

(b)

Fig. 4. (a) Terms required. (b) CPU time required for $0.01 \%$ convergence using accelerated spatial sum and unaccelerated spectral sum $D_{x}=0.1 \lambda, \phi^{\prime}=$ $30^{\circ}, y=0.1 \lambda$.

case. Where appropriate, asymptotic integral evaluation could dramatically reduce the required computation times.

Starting with the first component, we see the inverse bandwidth nature of the Fourier transform pairs determines the most efficient form. The key parameters are the interelement spacing and the location of the observation point. Generally, the spectral sum requires fewer terms than the spatial sum as the interelement spacing decreases and the observation point moves away from the array (either increasing positive values of $y$ or decreasing values of $x$, with $x<0$ ). In Fig. 3(a), we present the number of terms necessary to converge to the criteria

$$
\left|\frac{\text { Sum }- \text { Truth }}{\text { Truth }}\right|<1.0 \%
$$

where the "truth" value is taken from an extremely large number of terms (in either sum form). Note that along this observation path, the spectral sum requires only one term, a number far less than what the spatial sum requires.

Turning to the second component, one might ask, "Does the computational burden of calculating the continuous spectral integral negate any gains from the convergence advantage of the spectral domain form?" Fig. 3(b) answers this question for the subject test case by comparing the CPU time required to calculate the two summations on a Sparc10 workstation. Clearly, with only one term to calculate, the additional computational effort required for the spectral-domain sum is more than offset by the reduction in number of terms required for convergence. Of course, summation acceleration techniques such as Shank's transformation [13] or the Rho transformation [14] can be used to enhance the convergence of either the spatial or spectral sums. The data in Fig. 4 was generated

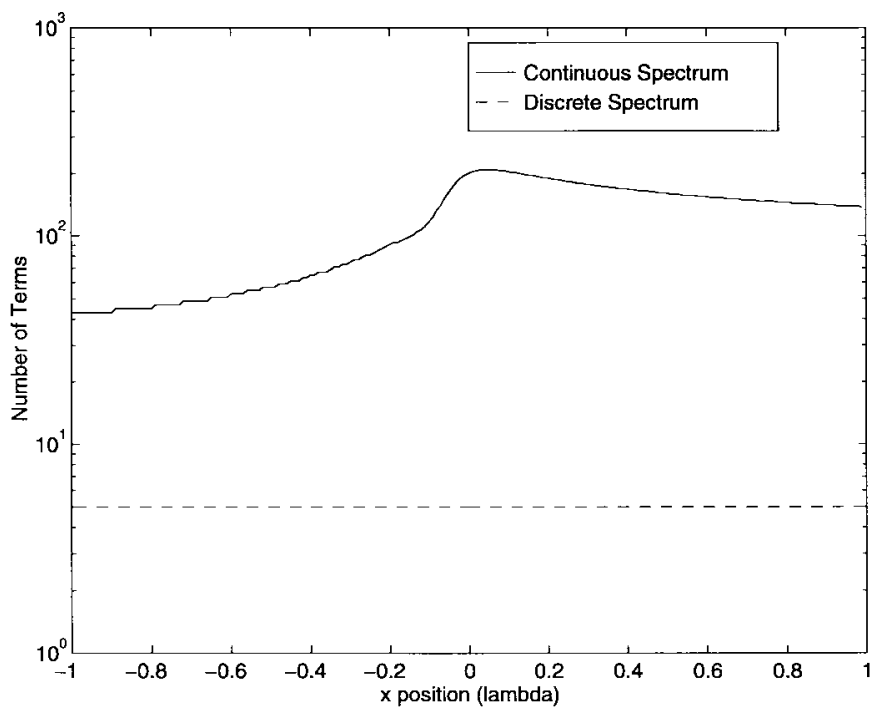

Fig. 5. Terms required for $0.0001 \%$ convergence in the discrete spectrum and the continuous spectrum sums $D_{x}=0.1 \lambda, \phi^{\prime}=30^{\circ}, y=0.1 \lambda$.

using a circle averaging acceleration scheme ${ }^{1}$ which significantly accelerates the spatial sum. The spectral sum was not accelerated. We've also tightened the convergence criteria to $0.01 \%$, causing the spectral sum to lose its computational advantage in some regions. We note, again, that aside from any computational advantage or disadvantage, the spectraldomain form is tremendously useful in problems containing a stratified dielectric media.

Another interesting phenomenon is the difference of the convergence rates for the discrete and continuous spectrum terms on the right-hand side of (9). Fig. 5 shows this convergence data along the inner two wavelengths $(-1 \lambda<x<+1 \lambda)$ of the observation path. This data was generated by forming the discrete spectrum sum (alone) and the continuous spectrum sum until the last term in each has changed the magnitude of the partial sum by no more than $10^{-6}=0.0001 \%$. This tends to be a tighter tolerance criteria than what was used for Figs. 3 or 4 . Note that the discrete spectrum is relatively insensitive to the $x$ position, whereas the convergence of the continuous spectrum changes dramatically along the path. Also note that the overall convergence rate of the spectral sum is dominated by the continuous spectrum terms, which we found to be generally true for other observation paths. Thus, it behooves us to use the asymptotic evaluation for $I_{n}$ as much as possible to maintain computational efficiency.

The accuracy of the asymptotic evaluation of the continuous spectrum integrals $I_{n}$ is driven by the magnitude of the large parameter $(\beta \tilde{\rho})$. Fig. 6 shows the dependence of the relative error between numerical and asymptotic evaluations of $I_{n}$ with $n=0$ as the large parameter takes on three distinct values. In this case, relative error is defined as

$$
\text { Relative Error }=\left|\frac{\text { Asymptotic }- \text { Numerical }}{\text { Numerical }}\right| \text {. }
$$

\footnotetext{
${ }^{1}$ The circle averaging scheme is based on the a priori knowledge that the sequence of partial sums in the spatial domain tends to form a slowly converging spiral in the complex plane.
} 


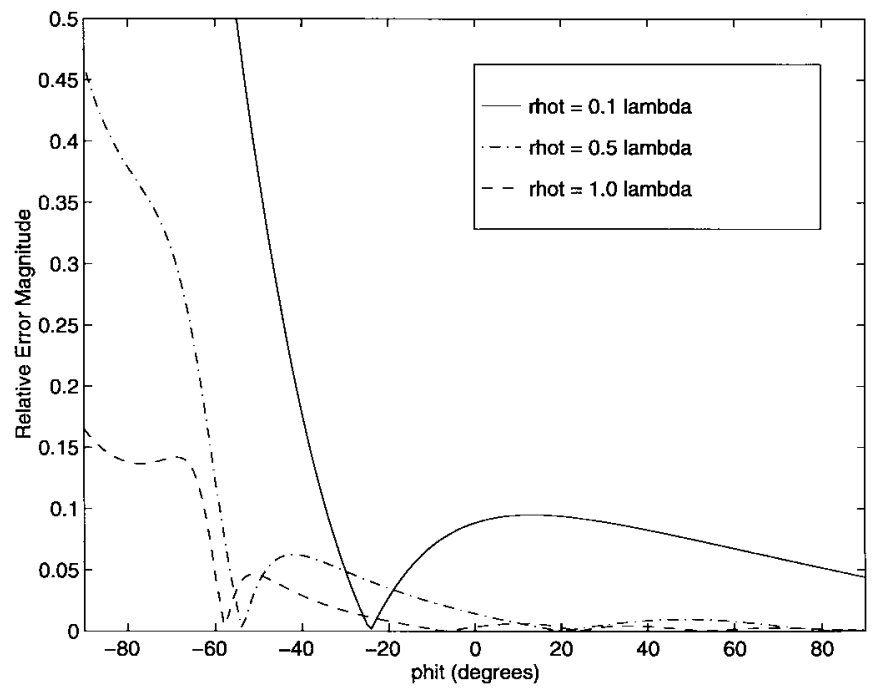

Fig. 6. Accuracy of the asymptotic evaluation of $I_{n}, n=0$ along angular sweeps in $\tilde{\phi}$ at fixed values of $\tilde{\rho}, D_{x}=0.1 \lambda, \phi^{\prime}=30^{\circ}$.

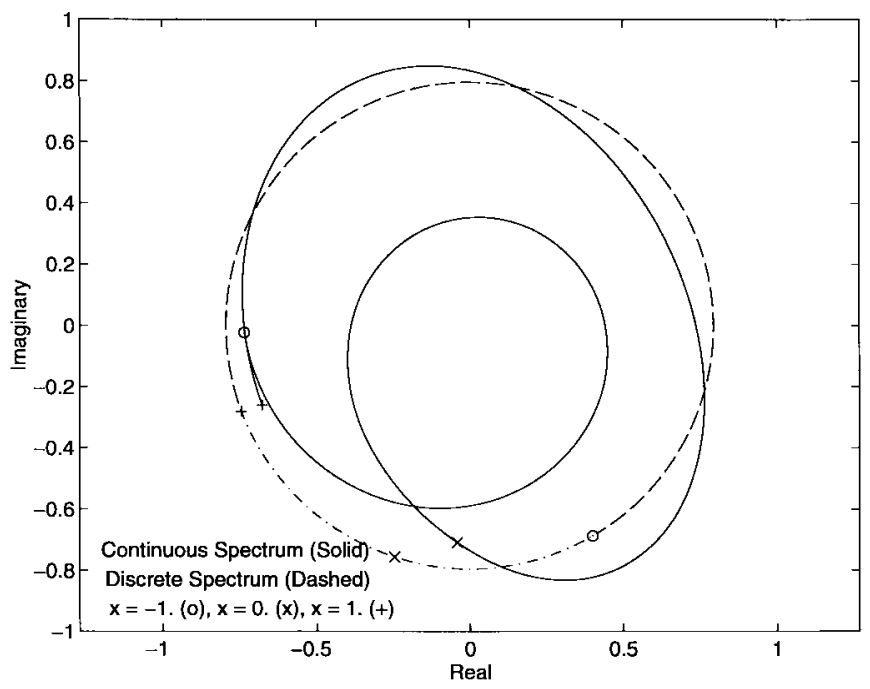

Fig. 7. Evaluation of the discrete spectrum sum and the continuous spectrum sum $D_{x}=0.1 \lambda, \phi^{\prime}=30^{\circ}, y=0.1 \lambda$.

For each $\tilde{\rho}$, the observation angle $(\tilde{\phi})$ is swept through an arc around the point $x=-\frac{D_{x}}{2}, y=0$. Note that the most difficult region for the asymptotic solution is the forward radiation sector $(\tilde{\phi}<0)$. This case $(n=0)$ corresponds to a propagating plane wave in the discrete spectrum, which tends to be the most challenging case for the asymptotic evaluation. For the evanescent modes, the large parameter can be reduced by at least an order of magnitude for a given acceptable error.

Finally, in Fig. 7 we present the evaluation of the Green's function in the complex plane as it is calculated from (9), split into the discrete and continuous spectrum terms. To prevent cluttering of the data, we truncate the observation path to $-1 \lambda<x<+1 \lambda$. Note that the evaluation from the discrete spectrum sum traces a nearly perfect circle (which is only so because the evanescent plane waves are almost negligible at $y=0.1 \lambda$ ), while the continuous spectrum sum is less predictable. Also note that the continuous spectrumsum magnitude is nearly identical to the discrete spectrum-sum magnitude at $x=+1 \lambda$ (data designated with plus symbols), and "in phase." This agrees with the understanding that as one observes the field "deep" over the array, the field nearly mimics the radiation of a fully infinite array that is exactly double the discrete spectrum of (9). On the other hand, the data from the two spectrums at $x=-1 \lambda$ (designated with the circles) is of similar magnitude, but strongly out of phase. Thus, the two spectrums are in a destructive interference to weaken the radiated field at negative values of $x$ as long as we have crossed the "shadow boundary" discussed in the SDP evaluation. In this example, with $D_{x}=0.1 \lambda, \phi^{\prime}=30^{\circ}$ and $y=0.1 \lambda$, the "shadow boundary" occurs at $x \approx-0.223 \lambda$.

\section{Conclusions}

A one-sided version of the Poisson sum formula was derived and presented in (5). It provides enhanced convergence in some applications, as a result of the inverse bandwidth relationship between Fourier transform pairs. It has been successfully applied to the problem of the radiation of a semiinfinite array of periodic line sources that obey Floquet's theorem, i.e., equal amplitude currents with a progressive phase factor. The result is a transformation of a spatial-domain sum to a spectral sum which contains both a discrete spectrum of plane waves and a continuous spectrum, which comes from a principal value integral. The continuous spectrum is evaluated either by numerical methods using a regularization of the integrand or by asymptotic analysis. Numerical results from each method are given here, which provide validation of the algebra and give insight into the radiation phenomena.

Numerical results show a significant reduction in the number of terms needed in the semi-infinite array free-space Green's function with the one-sided Poisson sum formula. An additional benefit from the transformation is that it puts the radiation purely in terms of plane waves, which allows for an extension to geometries involving infinite planar boundaries. As an example, we are currently working on applying the onesided Poisson sum formula for the problem of radiation of a semi-infinite array embedded in a dielectric slab (infinite in $x$ and $z$, but finite thickness in $y$ ). Such an extension introduces surface wave poles within the continuous spectrum integrals. In this case, the one-sided Poisson sum formula is necessary, as it would be extremely difficult to attack the array within a slab problem with a spatial-domain sum approach.

\section{ACKNOWLEDGMENT}

The authors would like to thank Dr. W. P. Baker at the Air Force Institute of Technology for many useful discussions on the various mathematical transformations we pursued.

\section{REFERENCES}

[1] L. B. Felsen and L. Carin, "Diffraction theory of frequency and timedomain scattering by weakly aperiodic truncated thin-wire gratings," $J$. Opt. Soc. Amer. A, vol. 11, pp. 1291-1306, Apr. 1994.

[2] L. Carin and L. B. Felsen, "Time harmonic and transient scattering by finite periodic flat strip arrays: hybrid (ray)-(Floquet mode)-(MOM) algorithm," IEEE Trans. Antennas Propagat., vol. 41, pp. 412-21, Apr. 1993.

[3] A. Papoulis, The Fourier Integral and Its Applications. New York: McGraw-Hill, 1962. 
[4] F. G. Stremler, Introduction to Communication Systems. Reading, MA: Addison-Wesley, 1977.

[5] J. D. Gaskill, Linear Systems, Fourier Transforms, and Optics. New York: Wiley, 1978.

[6] M. J. Lighthill, Introduction to Fourier Analysis and Generalized Functions. Cambridge: Cambridge Univ. Press, 1958.

[7] L. Brillouin, Wave Propagation in Periodic Structures, 2nd ed. New York: Dover, 1953.

[8] H. Bateman, Table of Integral Transforms. New York: McGraw-Hill, 1954, vol. I.

[9] B. A. Munk and G. A. Burrell, "Plane-wave expansion for arrays of arbitrarily oriented piecewise linear elements and its application in determining the impedance of a single linear antenna in a lossy halfspace," IEEE Trans. Antennas Propagat., vol. AP-27, pp. 331-43, May 1979.

[10] L. B. Felsen and N. Marcuvitz, Radiation and Scattering of Waves. Englewood Cliffs, NJ: Prentice-Hall, 1972.

[11] I. S. Gradshteyn and I. M. Ryzhik, Table of Integrals, Series, and Products. San Diego, CA: Academic, 1980.

[12] M. Abramowitz and I. E. Stegun, Eds., Handbook of Mathematical Functions with Formulas, Graphs, and Mathematical Tables. Washington, DC: U.S. Govt. Printing Office, 1972, U.S. Dept. Commerce, Nat. Bureau Standards Appl. Mathem. Ser.

[13] S. Singh, W. F. Richards, J. R. Zinecker, and D. R. Wilton, "Accelerating the convergence of series representing the free space periodic Green's function," IEEE Trans. Antennas Propagat., vol. 38, pp. 1958-1962, Dec. 1990.

[14] S. Singh and R. Singh, "On the use of the $\rho$-algorithm in series acceleration," IEEE Trans. Antennas Propagat., vol. 39, pp. 1514-1517, Oct. 1991.
J. Paul Skinner received the B.S. degree from Texas A\&M University, College Station, in 1982, the M.S.E.E. degree from the Air Force Institute of Technology, Wright-Patterson AFB, OH, in 1984, and the Ph.D. degree from The Ohio State University, Columbus, in 1991, all in electrical engineering.

From 1984 to 1988, he served a tour of duty at the Air Force Wright Aeronautical Laboratories, $\mathrm{OH}$, where he performed research in radar crosssection reduction. He is currently a Major in the United States Air Force, and Adjunct Associate Professor of electrical engineering at the Air Force Institute of Technology, Wright-Patterson AFB, OH.

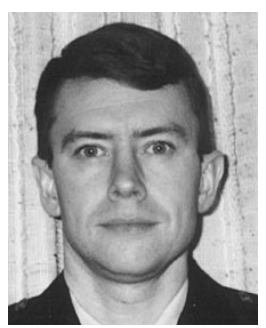

Peter J. Collins (S'93) received the B.S.E.E. and B.A. degrees from the University of Minnesota, Minneapolis-St. Paul and Bethel College, St. Paul, MN, respectively, both in 1985 , and the M.S.E.E. degree from the Air Force Institute of Technology, Wright-Patterson AFB, OH, in 1990. He is currently working toward the Ph.D. degree at the Air Force Institute of Technology.

From 1986 to 1989 , he served a tour of duty at the Air Force Wright Laboratories, $\mathrm{OH}$, where he performed research in electro-optic component development. After 1990 he served as Engineering Branch Chief with the Radar Target Scatter Division, 6585th Test Group, Holloman AFB, NM. His current research interests are in the area of computational electromagnetics and low observable technology. 